Iranian Journal of Pathology | ISSN: 2345-3656

\title{
Cutaneous Verrucous Carcinoma Superimposed on Chronically Inflamed Ileostomy Site Skin: A Case Report
}

\author{
Ali Yaghobi Joybari' ${ }^{1}$ Payam Azadeh${ }^{1}$, Behzad Nemati Honar ${ }^{2 *}$ \\ 1. Dept. of Radiation Oncology, School of Medicine, Shahid Beheshti University of Medical \\ Science, Tehran, Iran \\ 2. Dept. of Surgery, School of Medicine, Shahid Beheshti University of Medical Science, \\ Tehran, Iran
}

\begin{tabular}{c}
\hline KEYWORDS \\
\hline Verrucous; \\
Squamous Cell; \\
Cutaneous; \\
Carcinoma; \\
Ileostomy \\
\hline
\end{tabular}

\section{Article Info}

Received 25 Oct 2017; Accepted 10 March 2018; Published Online 17 July 2018;

\begin{abstract}
Verrucous carcinoma (VC) is a rare variant of well differentiated squamous cell carcinoma (SCC) which is usually found in oral cavity mucosa. Cutaneous verrucous carcinoma is a rare entity and in this paper we report a 43 years old man with VCsuperimposed on chronically inflamed skin of ileostomy site. Previously, he was operated to treat rectal adenocarcinoma and has had ileostomy for six months. The skin lesion was resected totally during surgical operation for ileostomy closure. Histopathologic examination confirmed the diagnosis of cutaneous verrucous carcinoma. Post-operative follow up shows no evidence of recurrence after six months. We suggest patient's training for follow up visits in order to early detection of osteomy site complications including neoplastic changes.
\end{abstract}

Corresponding information:

Behzad Nemati Honar, MD, Assistant Professor of Surgery, School of Medicine, Shahid Beheshti University of Medical Science, Tehran, Iran

Copyright (C) 2018, IRANIAN JOURNAL OF PATHOLOGY. This is an open-access article distributed under the terms of the Creative Commons Attribution-noncommercial 4.0 International License which permits copy and redistribute the material just in noncommercial usages, provided the original work is properly cited.

\section{Introduction}

Verrucous carcinoma (VC) is a rare variant of well differentiated squamous cell carcinoma (SCC) which described by Dr. Lauren Ackerman in 1948 for the first time $(1,2)$. VC is more prevalent in men and tends to present in elderly (3). This neoplasm is usually found in oral cavity mucosa. This low grade variant of SCC is also reported in pharyngeal, laryngeal, sinonasal and esophageal mucosa, etc. Cutaneous verrucous carcinoma (also known as Epitheliomacuniculatum) is much rarer entity and was reported in head and neck, palmoplantar, anogenital and gluteal skin, etc (2). There are evidences supporting the role of tobacco use and Human papilloma virus (HPV) as predisposing factors for developing $\mathrm{VC}(4,5,6)$. Indolent cytological appearance paradoxical to the local invasion potential makes a unique neoplastic character for $\mathrm{VC}^{2,7}$. Although cases of lymph node metas- tasis were reported rarely, tendency of VC for distant metastasis is dispensable $(1,2,8)$.

In this paper we report a case of VC superimposed on chronically inflamed skin of ileostomy site.

\section{Case Report}

A 43 years old smoker man presented with painless rectorrhagia for 2 months and referred to gastrointestinal specialist for further evaluation. In his colonoscopy a rectal mass was seen $7 \mathrm{~cm}$ distant from anal sphincter. Biopsy was taken and sent for histopathologic examination which confirmed the diagnosis of adenocarcinoma. In preoperative work up, there was no evidence of distant metastasis. After accomplishing neo-adjuvant chemotherapy, he was admitted for surgical treatment (ultra-low anterior colon resection and protective loop ileostomy). Post-operative course was uneventful and chemotherapy was continued be- 
fore he underwent the second surgical operation for anastomosis of bowel and ileostomy closure. The skin ring harboring the open loop of small bowel was resected and sent for pathologic evaluation.

In gross pathologic examination of the specimen presence of multiple grayish white exophytic non ulcerated skin warty lesions was remarkable. The lesions were polypoid with filiform superficial projections and broad area of attachment to the underlying skin (Figure 1).

Microscopic assessment of the Hematoxylin and Eosin (H\&E) stained slides confirmed the diagnosis of VC for the skin lesions which resected completely with free surgical margins (Figure 2). Follow up of the patient for six months did not show any evidence of recurrence.

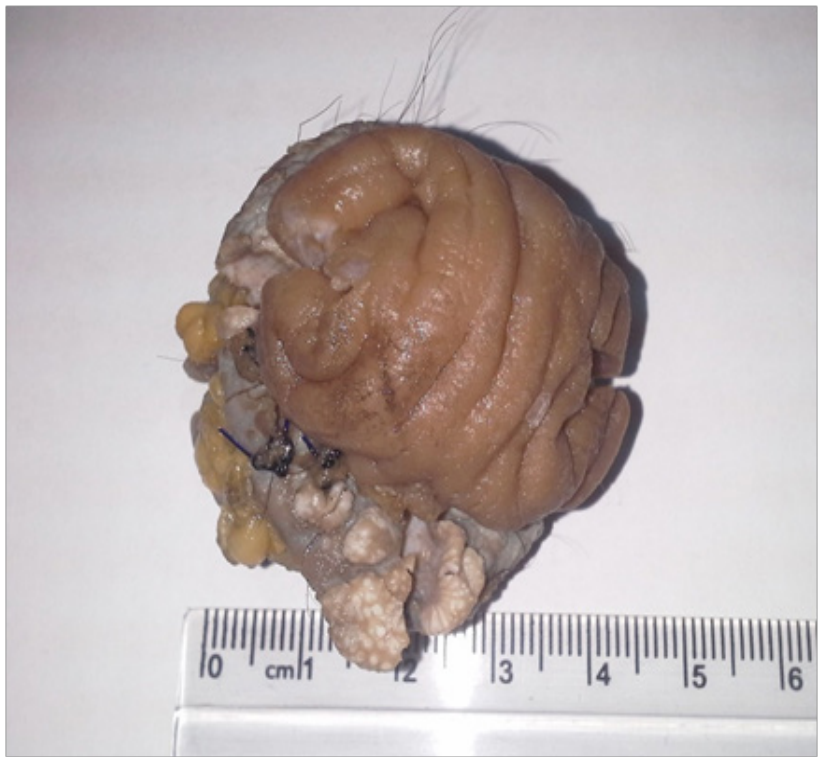

Figure 1. Cutaneous verrucous carcinoma of ileostomy site in gross pathologic examination shows polypoid lesion with verruca-like surface
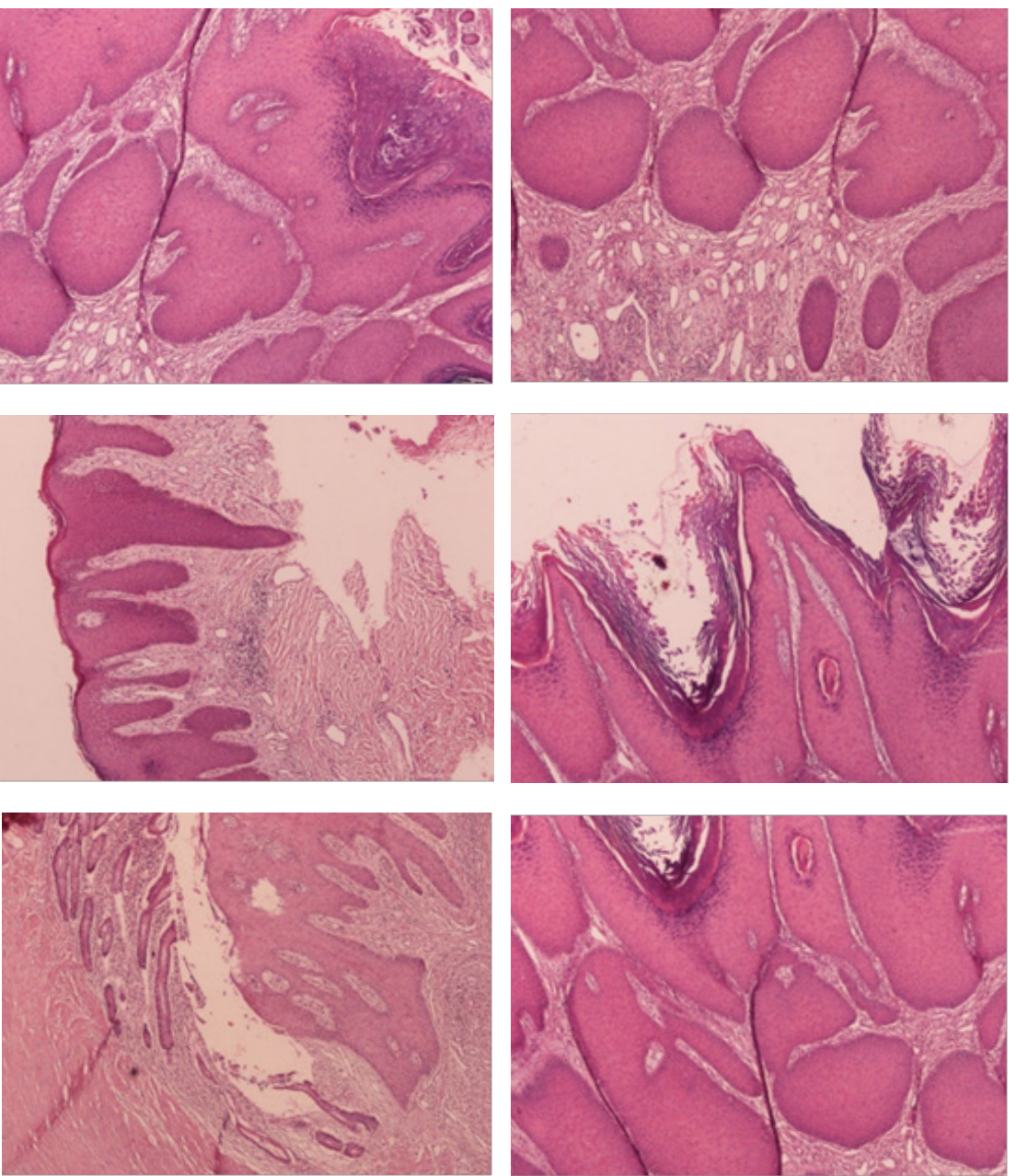

Figure 2. Cutaneous verrucous carcinoma of ileostomy site in microscopic histopathologic examination reveals verruca type hyperkeratosis and papillomatosis at surface and endophytic growth of elephant feet-like rete pegs deep into the stroma with reactive dermal chronic inflammation. Lack of squamous cell atypia and intactness of basal membrane are also notable. (Hematoxylin and Eosin staining $\times 25$ ) 


\section{Discussion}

Cutaneous verrucous carcinoma is a rare variant of well differentiated squamous cell carcinoma with local invasion potential. Grossly, it appears as a soft papillary fungating skin lesion invades contagious structures, slowly. Microscopically, verruca type acanthosis and hyperkeratosis plus benign looking papilomatosis could be seen on superficial areas while broad expansion of squamous rete pegs could be seen in deeper areas with club shape pushing borders $(1,2,8)$.

Differential diagnoses for VC could be simple wart and invasive types of well differentiated squamous cell carcinoma.

On one hand and in gross examination of the present specimen, the lesions consist of non-ulcerative white gray soft nodules with cauliflower-like surface which could be in differential diagnosis with verruca vulgaris (simple wart). At microscopic level, pushing borders of elephant feet-like endophytic projections confirm the nature of the neoplasm and exclude verruca vulgaris as a differential diagnosis. On the other hand, other variants of squamous cell carcinoma should be ruled out as differential diagnoses. Morphologically, absence of atypia and non-infiltrating borders of the lesion could be strong clues to rule out the later differential diagnoses.

As a matter of fact, site of surgical ostomies may develop various complications by time such as stenosis, irritation, ulceration, herniation, etc. Neoplastic changes in ileostomy site are quite rare and reports of cases with squamous cell carcinoma of ileostomy sites are extremely rare, worldwide. Most of the ileostomy associated neoplastic lesions develops during long periods of time (2-24 years). Interestingly in this case, VC superimposed on ileostomy associated chronic dermatitis merely after 6 months. Increased risk of developing conventional types of SCC on chronically injured and irritated skin is quite clear. Thus, chronic mechanical and chemical damage of skin could be considered as possible contributory factors for development of $\mathrm{VC}$, too.

It seems to be logic to suggest regular follow up plan for ostomy sites in order to diagnose related complications earlier.

\section{Conclusion}

Cutaneous VC is rare variant of SCC and VC of ileostomy site is an extremely rare condition. Chronic mechanical and chemical damage to the skin of ileostomy site could be considered as possible contributory factors. More studies are required to proof and/ or explain their potential roles in development of VC. Early detection of the ostomy sites complications is possible simply by regular follow up visits. We suggest encouraging the patients for regular visits following ostomy surgeries in order to detect complications earlier, especially potential neoplastic changes.

\section{Conflict of Interest}

The authors declare no financial relationship with any organization regarding this research.

\section{References}

1. Ackerman LV. Verrucous carcinoma of the oral cavity. Surgery. 1948;23:670-8. PMID : $\underline{18907508}$

2. Rosai J. Rosai and Ackerman's Surgical Pathology. 10nd ed. Amsterdam: Elsevier; 2011. P. 246

3. Santoro A, Pannone G, Contaldo M, Sanguedolce F, Esposito V, Serpico R, et al. A troubling diagnosis of verrucous squamous cell carcinoma ("the bad kind" of keratosis) and the need of clinical and pathological correlations: a review of the literature with a case report. J Skin Cancer. 2011;2011:370605. https:// doi.org/10.1155/2011/370605 PMID:21151509 PMCID:PMC2989749

4. Brandsma JL, Steinberg BM, Abramson AL, Winkler B. Presence of Human Papillomavirus Type 16 Related Sequences in Verrucous Carcinoma of the Larynx. Cancer Res. 1986;46(4 Pt 2):2185-8. PMID:3004723

5. Fujita S, Senba M, Kumatori A, Hayashi T, Ikeda T, Toriyama K. Human papillomavirus infection in oral verrucous carcinoma: genotyping analysis and inverse correlation with p53 expression. Pathobiology. 2008;75(4):257-64 https://doi.org/10.1159/000132387 
6. Walvekar RR, Chaukar DA, Deshpande MS, Pai PS, Chaturvedi P, Kakade A, et al. Verrucous carcinoma of the oral cavity: a clinical and pathological study of 101 cases. Oral Oncol. 2009;45(1):47-51. PMID: $\underline{18620896}$

7. Oliveira DT, de Moraes RV, Fiamengui Filho JF, Fanton Neto J, Landman G, Kowalski LP.
Oral verrucous carcinoma: a retrospective study in São Paulo Region, Brazil. Clin Oral Investig. 2006;10(3):205-9. https://doi.org/10.1007/ s00784-006-0050-7 PMID: $\underline{16738900}$

8. Spiro RH, Verrucous carcinoma, then and now. Am J Surg. 1998;176(5):393-7. https://doi. org/10.1016/S0002-9610(98)00232-3 